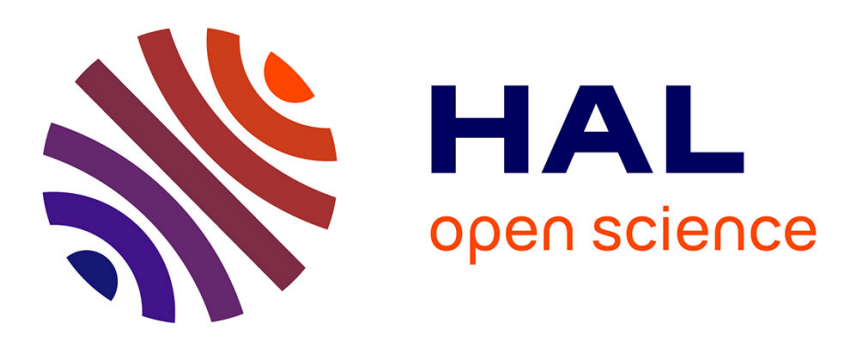

\title{
Effects of Mixtures of Alkanes on the Bending Rigidity Constant K of AOT Monolayers at the Planar Oil-Water Interface
}

S. Chaieb, B. Binks, J. Meunier

\section{> To cite this version:}

S. Chaieb, B. Binks, J. Meunier. Effects of Mixtures of Alkanes on the Bending Rigidity Constant K of AOT Monolayers at the Planar Oil-Water Interface. Journal de Physique II, 1995, 5 (9), pp.1289-1296. 10.1051/jp2:1995184 . jpa-00248235

\section{HAL Id: jpa-00248235 https://hal.science/jpa-00248235}

Submitted on 1 Jan 1995

HAL is a multi-disciplinary open access archive for the deposit and dissemination of scientific research documents, whether they are published or not. The documents may come from teaching and research institutions in France or abroad, or from public or private research centers.
L'archive ouverte pluridisciplinaire HAL, est destinée au dépôt et à la diffusion de documents scientifiques de niveau recherche, publiés ou non, émanant des établissements d'enseignement et de recherche français ou étrangers, des laboratoires publics ou privés. 
Classification

Physics Abstracts

$6220 \mathrm{Dc}-64$ 60-1 $-68.10-\mathrm{m}$

\title{
Effects of Mixtures of Alkanes on the Bending Rigidity Constant K of AOT Monolayers at the Planar Oil-Water Interface
}

\author{
S. Chareb $\left({ }^{1}\right)$, B. P. Binks $\left({ }^{2}\right)$ and J. Meunier $\left({ }^{1}\right)$ \\ $\left({ }^{1}\right)$ Laboratore de Physıque Statıstıque de l'Ecole Normale Supéneure, 24 rue Lhomond, 75231 \\ Paris Cedex 05, France \\ $\left({ }^{2}\right)$ School of Chemistry, Unversity of Hull, Hull HU6 7RX, UK
}

(Received 6 April 1995, received in final form 7 June 1995, accepted 12 June 1995)

\begin{abstract}
We have studied the phase diagrams of brine/oil/AOT mixtures in which the oils are mixtures of octane and tetradecane and the amount of AOT is small In addition, the properties of the AOT monolayer at the brine/oll interface as a function of the volume fraction $\phi$ of tetradecane in the oll have been measured. The values of the interfacial tension and the phase diagram behaviour show that the Equrvalent Alkane Carbon Number concept is valid in these systems, where a small change of $\phi$ induces a large change in the phase diagram. In addition we show that this concept applies to the bending elastic modulus $K$ and probably to the saddle splay modulus $\bar{K}$
\end{abstract}

The phase diagram of mixtures containing the ionic surfactant AOT (sodium diethylhexysulphosuccinate), brine and an alkane has been studied [1,2]. For low AOT concentrations but in excess of that required for aggregation (c.m.c., i.e., critical micellar concentration), high salt concentration $(S)$ results in water-in-oil (w/o) microemulsions coexisting in equilibrium with excess aqueous phase. At low $S$, systems are formed in which an AOT-rich phase coexists with excess oil phase (and sometımes with excess aqueous phase). At intermediate $S$, three phases may form for which a surfactant-rich phase (lamellar or isotropic) coexists with both excess water and oil phases. By analogy with the Winsor equilibria [3], these three different equilibria have been called Winsor II, Winsor I and Winsor III systems respectively. However, the AOT rich phase in the Winsor III equilbrium is not the common bicontinuous phase and the Winsor I equilibrium is more complicated than that observed in the true Winsor systems. The mechanical properties of the AOT monolayer at the alkane-water interface were investigated in previous work for various pure $n$-alkanes $[4,5]$. The bending elastic constant $K$ and the saddle splay elastic constant $\bar{K}$ are strongly oll dependent. On the one hand, $K$ is large $\left(\sim k_{\mathrm{B}} T\right)$ with short chain alkanes (carbon number $N<11)$ and is small $\left(\sim 0.1 k_{\mathrm{B}} T\right)$ with long chain alkanes $(N>11)$. A strong jump in the $K$ modulus is observed when $N$ increases from 10 to 12 [4]. On the other hand, in the middle of the Winsor III systems, when the spontaneous curvature of the AOT monolayer vanishes, $\bar{K}$ is negative for short chain alkanes and positive 
for long chain alkanes [5]. The difference in the nature of the AOT-rich phase in the Winsor III equilibria obtained with short chain alkanes or with long chain alkanes results in the difference in the mechanical properties of the AOT monolayer depending on the length of the alkane [6]. These differences in the mechanical properties are related to the differences in solubilization of alkanes into the AOT tails which is different for short and long oils [7,8]. As a consequence of previous work, a monolayer of a single pure surfactant as AOT at an oil water-interface has to be considered as a mixed film: the aliphatic region of the AOT monolayer is composed of a mixture of its own paraffinic tails and the molecules of the $n$-alkane, the amount and the mobility of which vary depending on whether the $n$-alkane is a "good" or a "poor" solvent for the aliphatic tails [8].

The present paper is concerned with an AOT monolayer at an oil-water interface where the oil is a mixture of a short chain alkane (octane, $N=8$ ) and a long chain alkane (tetradecane, $N=14$ ). Firstly, we are interested in a more precise analysis of the role of the film elastic constant in the determination of the phase structure. Indeed, in the case of alkane mixtures, a continuous variation of the mechanical properties of the film with oll composition is expected. Secondly, it is important to understand the effect of alkane mixtures on the film properties for practical applications where frequently alkanes are mixtures. Thirdly, we show that the equivalent alkane carbon number (EACN) concept [9] inferred from phase diagram studies and interfacial tension measurements is valid for the bending elastic modulus of the monolayer. We have studied the equilibrium phase behaviour at $20^{\circ} \mathrm{C}$ of these systems, dilute in AOT, as a function of $S$, the salt concentration of the brine, for various volume fractions $\phi$ of tetradecane in octane ranging from $5 \%$ to $80 \%$. Typically, equal volumes of alkane mixtures and AOT solution in brine were equilibrated in a thermostat until phase separation was complete. In general, the phase separation is much longer than with pure alkanes and poor on a reasonable time scale (poorer than with pure alkanes), particularly at low $S$, before the Winsor III range and at low volume fractions of tetradecane, probably because the Ostwald ripening that breaks the emulsions is slower [10]. Consequently, the determination of the phase diagram is difficult and not as accurate as for pure alkanes. However, the phase diagrams with alkane mixtures that we have obtained have similarities with those obtained with pure alkanes. For low $\phi$ ( $\phi \lesssim 30 \%$ ), the Winsor III domain has a narrow range in salinity. A lamellar (birefringent) phase coexists with the oil phase and the aqueous phase as for pure octane at the lowest $\phi$. At higher $\phi$, and for the highest salinities, the lamellar phase coexists with an excess of oil and brine, while at the lowest salinities the lamellar phase incorporates all the brine. In this case, where there is no excess of brine, one can observe the coexistence of two different lamellar phases: Figure 1a for $\phi=0.25$. For high volume fractions of tetradecane $(\phi \gtrsim 50 \%)$, the longer alkane phase behavior dominates. At low salinities and very low AOT concentrations, an isotropic phase coexists with an excess of oil. At higher AOT concentrations a birefringent phase coexists with the oil phase as in the phase diagram obtained with pure dodecane. When the salinity $S$ is increased, an isotropic phase appears. Its volume increases with $S$ and it occupies the total volume of the aqueous phase (Winsor I equilibrium). Increasing $S$ further, a three-phase equilibrium appears (Winsor III). A phase rich in AOT appears at the bottom of the tube, in equilibrium with an excess of brine (in which the AOT concentration is equal to the $\mathrm{cmc}$ ) and oil. The volume of the rich AOT phase decreases and is at its minimum close to the optimal salinity $S_{0}$ (the salinity at which the AOT film has zero spontaneous curvature, see below) (Figs. 1c and $1 \mathrm{~d}, \phi=0.5$ and $\phi=0.6$, respectively). At high salinities the rich AOT phase is the oil phase (Winsor II equilibrium). At intermediate $\phi(\phi=0.4$, Fig. 1b), we have observed the same behavior, except in the optimal salnity region where a four-phase equilibrium appears, the fourth phase is a small drop of a birefringent phase at the bottom of the tube, coexisting with the oil phase, the aqueous phase and the isotropic AOT-rich phase 

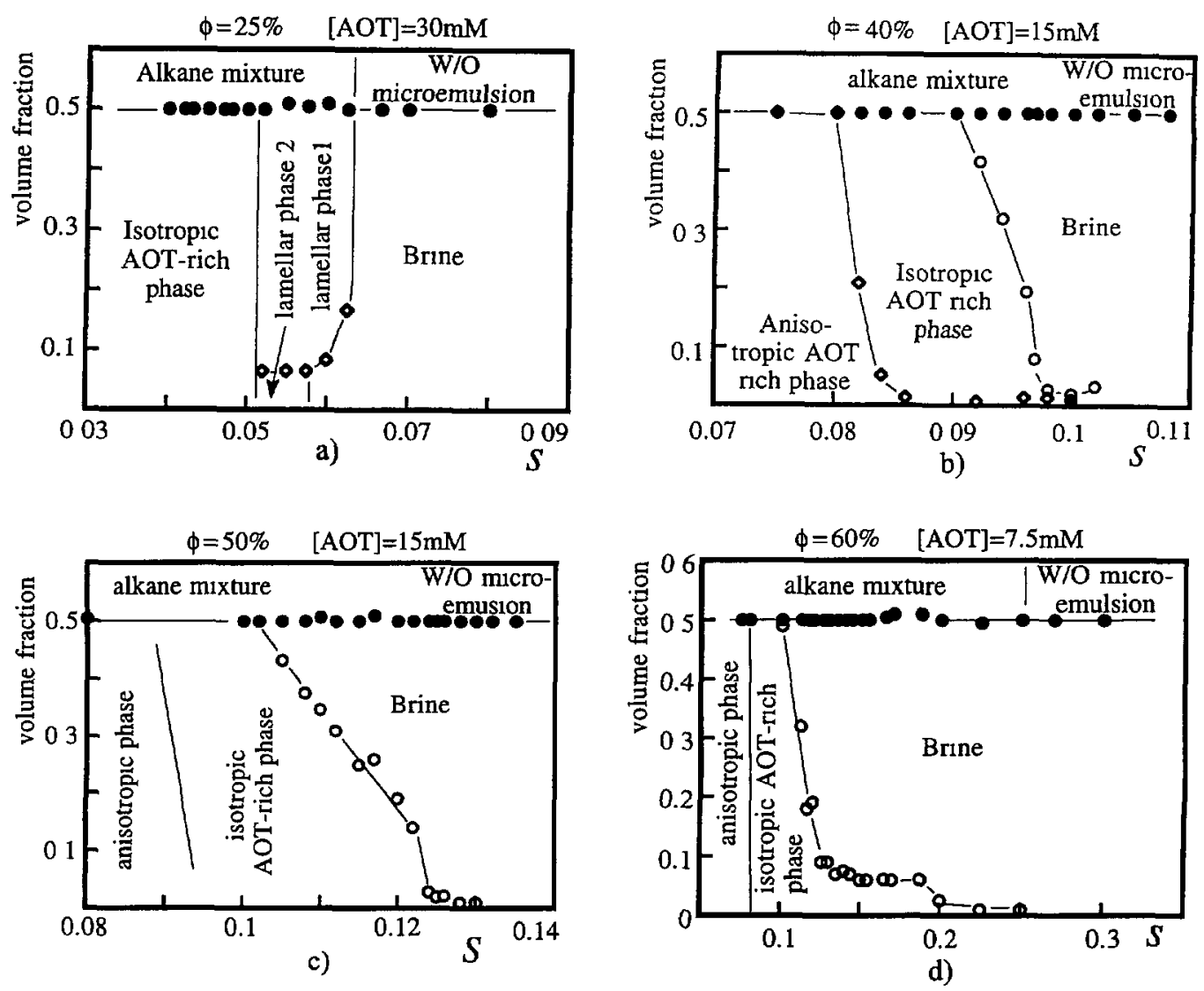

Fig 1 - Fraction of the total volume (in ordinate) occupied by the different phases versus the salinity $S$ for different volume fractions $\phi$ of tetradecane in the oll Measurements are for a mixture of brine (50\% in volume) and octane/tetradecane/AOT (50\% in volume) at $20{ }^{\circ} \mathrm{C}$ a) $30 \mathrm{mM}$ AOT in the alkane mixture with $\phi=25 \%$ of tetradecane, b) $15 \mathrm{mM}$ AOT in the alkane mixture $\phi=40 \%$ of tetradecane, c) $15 \mathrm{mM}$ AOT in the alkane mixture $\phi=50 \%$ of tetradecane, d) $7.5 \mathrm{mM}$ AOT in the alkane mixture $\phi=60 \%$ of tetradecane In this representation, the total volume is 1 , but we have limited the ordinate to the range $0-0.6$ because above approximately 0.5 the phase rich in oil is homogenous

(Fig. 1b). It could be due either to a partial and slight separation of the two alkanes or to a non-equilibrium state. It must be remarked that, for practical reasons, the AOT concentration was decreased for increasing $\phi$ in the phase diagrams of Figures 1a-d: for low $\phi$, the volume of the lamellar phase becomes too small to be measured with sufficient accuracy at low AOT concentration and, at large $\phi$, the equilibration time increases very quickly with the AOT concentration. However, we did observe that a variation of the AOT concentration does not change the general features presented here. It produces only a slight change of the salinities at which the phase transitions take place.

Oil-water interfacial tension measurements have been used in conjunction with interfacial ellipticities to determine the rigidity $K$ of the surfactant monolayer. Interfacial tensions between 


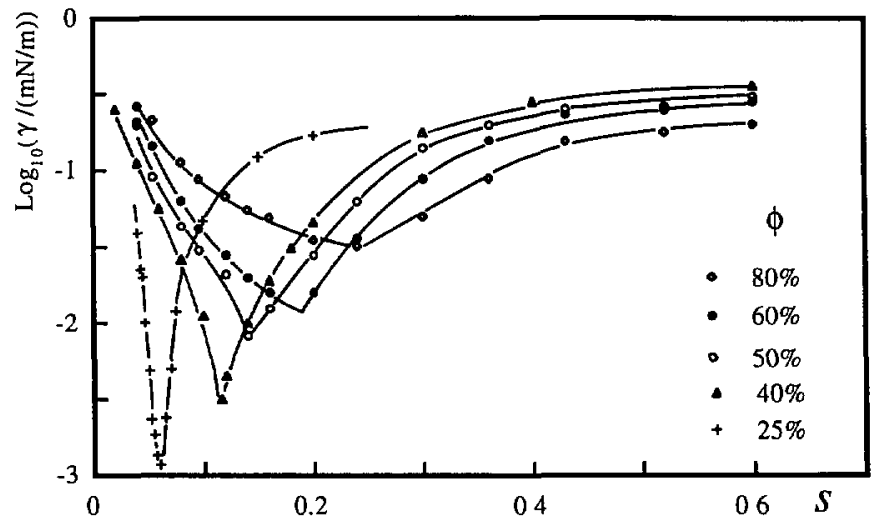

Fig. 2 - Variation of the post - c m.c oil - water interfacial tension with the molar salinity of the water, $S$, at $20^{\circ} \mathrm{C}$ for AOT for different volume fractions of tetradecane in octane The [AOT] in the systems is $75 \mathrm{mM}$.

equilibrium phases were measured using a Kruss Site 04 spinning drop tensiometer. Interfacial ellipticity measurements were performed on a homemade ellipsometer, mounted vertically to reflect off the horizontal liquid surface [11]. In three-phase systems, both the tension and the ellipticity refer to the interface between excess ol and aqueous phases (brine), measured at $20^{\circ} \mathrm{C}$. These two phases are superposed in the ellipsometric cell and a drop of third AOT-rich phase is added which maintains equilibrium in spite of a small temperature change $\left(0.1{ }^{\circ} \mathrm{C}\right)$ or AOT adsorption on the walls of the cell. This drop does not wet the oll/aqueous interface which is only covered with an AOT monolayer, the subject of our study. The refractive indices of the bulk phases were determined using an Abbé refractometer.

Increasing the salinity from low salınities, the interfacial tension $\gamma$ decreases to a minimum $\gamma_{0}$, reached at the salinity $S_{0}$ then increases for higher salinities (Fig 2) $S_{0}$ is the "optimal salinity" and is the concentration of $\mathrm{NaCl}$ required to phase invert microemulsions of AOT (close to the middle of the Winsor III salt range), or the salinity at which the spontaneous curvature of the AOT monolayer vanıshes. Upon increasing $\phi$, the volume fraction of tetradecane, $S_{0}$ and $\gamma_{0}$ increase (Fig. 2). However, at low tetradecane concentration ( $\phi \lesssim 30 \%$ ), the optimal salinity is constant and only the minumal interfacial tension $\gamma_{0}$ increases.

The ellipsometric parameter $\eta$ is derived from the measured ellipticity $\bar{\rho}$ of an interface and the dielectric constants at light frequency $\left(\epsilon_{1}, \epsilon_{2}\right)$ of the two bulk phases. It depends upon the interfacial properties and is the sum of two terms. $\eta^{\mathrm{R}}$ due to the surface roughness and $\eta^{l}$ due to the surface thickness and anisotropy. In the case of a thin but rough interface [11],

$$
\eta=\eta^{l}+\eta^{\mathrm{R}}=\eta^{l}-\frac{3 k T}{4 \pi \gamma} \frac{\left(\epsilon_{1}-\epsilon_{2}\right)^{2}}{\left(\epsilon_{1}+\epsilon_{2}\right)} q_{m}, \quad \text { where } q_{m}=\frac{\pi}{2} \sqrt{\frac{\gamma}{K}}
$$

where $q_{m}$ is an upper cut-off for the capillary waves resulting from the curvature energy of the interfacial film. By combining the two expressions, it is clear that if $K$ is constant, a plot of $\eta$ vs $\left(\epsilon_{1}-\epsilon_{2}\right)^{2} /\left[\left(\epsilon_{1}+\epsilon_{2}\right) \gamma^{05}\right]$ should be linear, the slope of which yields information on $K$, and the intercept on the $y$-axis is $\eta^{l}$ It was previously noted that for a given system in which only the salinity is varied, the measured elliptıcities are the same for two values of 


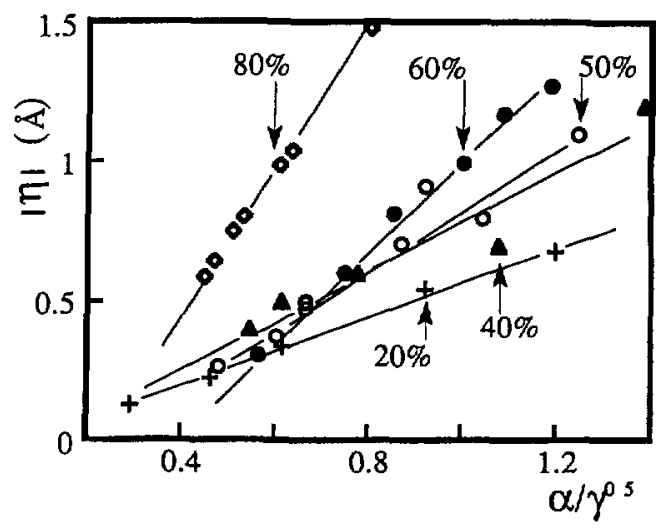

Fig 3 - The ellipsometric parameter $\eta$ vs $\frac{\left(\epsilon_{1}-\epsilon_{2}\right)^{2}}{\left(\epsilon_{1}+\epsilon_{2}\right) \gamma^{05}}=\frac{\alpha}{\gamma^{05}}$ for AOT monolayers in brine/octane-tetradecane mixtures at $20^{\circ} \mathrm{C}$ for different values of the volume fraction $\phi$ of tetradecane in the alkane mixture

the salınity where the values of $\gamma$ are equal $[12,13]$, indicating that, within the experimental accuracy, only $\gamma$ and not $K$ is modified with the salinity. In the following, this result was used and not systematically tested on these new systems. Most of the ellipsometric measurements were performed at high salinities, $S \gtrsim S_{0}$, because it was difficult and it took a long time to get good phase separation and consequently a clean oil/water interface at lower salinities. We show such plots in Figure 3 for different volume fractions of tetradecane. Within the error of the measurements, these plots are linear and the slopes of the lines increase as the volume fraction of the longer oil increases. This means that the bending elastic constant $K$ decreases with $\phi$. The best fit straight lines to the data in Figure 3 yield the values of $K$ shown in Figure 4. A large scatter of the $K$ values is observed at low $\phi$, i.e., at large $K\left(K \sim k_{\mathrm{B}} T\right)$ and results from the difficulty in obtaining a clean oil/water interface with a relatively rigid interface. This is no longer the case at higher concentrations ( $\phi \gtrsim 40 \%)$. Figure 4 suggests that for $\phi$ up to $25 \%$, the bending elastic constant is virtually constant at approximately $1 k_{\mathrm{B}} T$, and then falls steadily when $\phi$ increases above this volume fraction, reaching a value of $0.065 k_{\mathrm{B}} T$ for $\phi=80 \%$.

The equivalent alkane carbon number (EACN) concept has been developed for industrial applications from empirical mixing rules deduced from the phase behavior and the oil/brine interfacial tension in the presence of surfactants. It is important for practical applications because it is a very simple rule that allows predictions of the behavior of brine/oil/surfactant mixtures in cases where the oil is a complicated mixture. It allows one to reduce the behavior of these systems to the behavior of the same system in which a pure alkane takes the place of the complicated mixture.

Salager et al. [9] have shown that the optimal salinity (i.e., the salinity at which the oil/water interfacial tension is minimum) and the value of this minimum interfacial tension of an oil mixture/water interface are those measured at a pure alkane/water interface in which the length of the pure alkane is given by the equivalent alkane carbon number (EACN) concept. Assuming the ideality of the octane/tetradecane mixture, $N$ is linearly related to the volume 


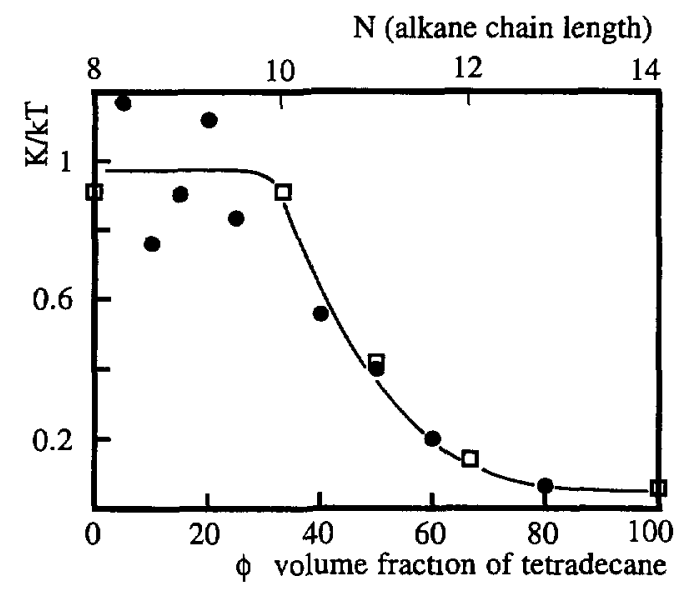

Fig. 4 - Variation of the bendıng elasticity constant $K$ in units of $k T$ versus $\phi$, the volume fraction of tetradecane in the alkane mixture and versus the equivalent alkane carbon number of the mixture, $N$. The $K$ values measured with pure alkanes [4] are reported on the graph as squares.

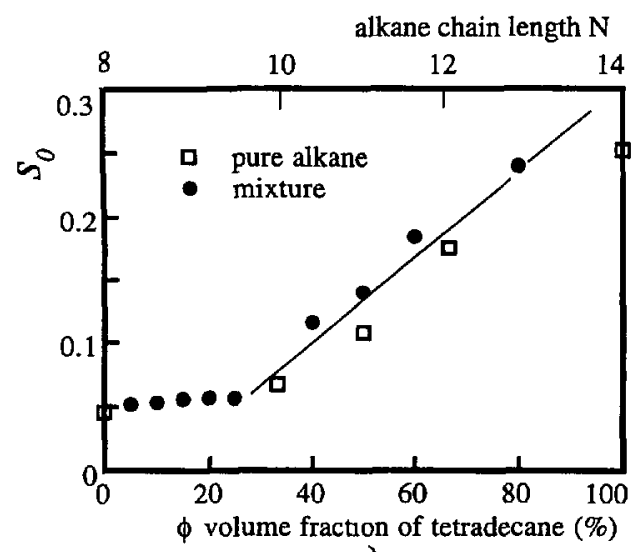

a)

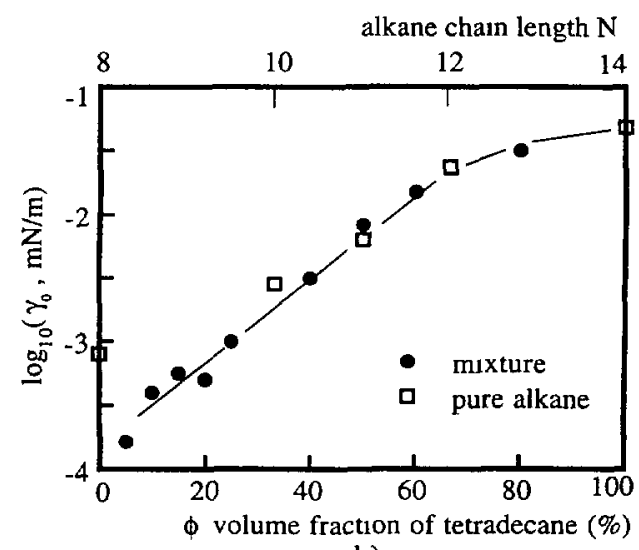

b)

Fig. 5 - a) Optimal salinitıes, $S_{0}$; b) munmal interfacial tension, $\gamma_{0}$, (on a logarithmic scale) versus $\phi$, the volume fraction of tetradecane and versus the equivalent alkane carbon number, $N$, of the mixtures. The $S_{0}$ and $\gamma_{0}$ measured with pure alkanes are reported on the same graphs

fraction of tetradecane, $\phi$ :

$$
N=14 \phi+8(1-\phi)
$$

In Figures $5 \mathrm{a}$ and $5 \mathrm{~b}$ we have plotted the optımal salinity and the logarithm of the minimum interfacial tension respectively versus the tetradecane volume fraction. On the same graphs, we have indicated the corresponding value $N$ of the EACN and we have plotted the optimal salinitıes and the minımum interfacial tension for pure alkanes. The optimal salinities and the 
logarithm of the minimum interfacial tension for pure alkanes and alkane mixtures are on the same lines within the experimental accuracy.

In Figure 4, where the bending elastic constant $K$ of the AOT monolayer at the brine octane/tetradecane mixture interface is plotted versus the tetradecane volume fraction, the value $N$ of the EACN (equivalent carbon number of the alkane mixture) has been indicated. On the same graph, we have plotted the $K$ values for AOT monolayers at pure alkane/water interfaces versus the alkane chain length $N$ for $8 \leq N \leq 14$ [4]. We observe that the $K$ values for pure alkanes and for mixtures are located on the same line with a good accuracy, suggesting that the EACN concept is valid for the the bending elastic modulus $K$ of surfactant films at oil/water interfaces. However, it has been tested with only one particular two-component mixture (octane/tetradecane). More investigations would be necessary to confirm the generality of this concept applied to the bending elasticity. However, the EACN concept applies to phase diagrams and to oil/brine interfacial tensions in the presence of surfactants. As $K$ is related to the interfacial tension and to the phase diagram (for instance through the radius of the droplets in the Winsor I and II regions), one can expect that this result can be extended to complicated mixtures.

$K$ is related to the quality of the alkane solvent for the AOT tails [8]; short chain alkanes $(N<11)$ are "good" solvents for the AOT tails while long chain alkanes are "poor" solvents for AOT tails. The validity of the EACN concept implies that, in these systems, on the one hand an alkane mixture behaves at least to a first approximation as a single pseudocomponent, and on the other hand, the solvent quality of this mixture for the surfactant tails is that of an $n$-alkane, the number of carbon atoms of which is given by the EACN concept, i e., equation (2) or a generalisation of this equation in the case of a more complicated mixture [9]. Do these results imply that there is no partitioning of the different oils penetrating in the monolayer? This is not necessarily the case; it could be that only the octane penetrates the monolayer but that the amount of octane in the monolayer depends upon the ratio $\phi$ of tetradecane outside the monolayer. We will undertake NMR measurements to decide between the different hypotheses.

On the one hand, the bending elasticity $K$ of the AOT monolayer was found to be only a function of the EACN, $N$. On the other hand, the phase diagrams with pure alkanes or mixtures we have studied appear, at least to a first approximation, to be only a function of the EACN. This means that the saddle splay constant, $\bar{K}$, is probably also only a function of the EACN because $K$ and $\bar{K}$ are the two elastic constants that determine the phase structure in the Winsor III region of the phase diagram, the region where the AOT monolayer which separates the oil and the brine micro-domains has a zero spontaneous curvature.

In conclusion, we have measured the interfacial tension $\gamma$ and the bending elastic modulus $K$ of an AOT monolayer at an octane-tetradecane mixture/brine interface versus the volume fraction of tetradecane. We have also studied the phase diagrams of octane-tetradecane-brine mixtures with a small amount of AOT. We have concluded that the equivalent alkane carbon number (EACN) concept applies to the $K$ modulus and probably to the Gaussian modulus $\bar{K}$. On the one hand, this concept can be used to study in detail the transition between lamellar phases and bicontinuous phases that is observed when the alkane chain length of the alkane increases, varying $N$ continuously. On the other hand, if it is confirmed that this result can be generalised for any oil mixture, it could be an interesting way to study the effect of very short alkanes on the elastic constants of the AOT monolayers. Indeed, very short chain alkanes are gaseous at atmospheric pressure; a short chain liquid alkane $(N \sim 6-8)$ mixed with benzene $(N=0)$ could be put in place of such gaseous alkanes. 


\section{References}

[1] Ghosh O. and Miller C A., J. Phys. Chem. 91 (1987) 4528

[2] Kellay H., Hendrikx Y., Meuner J., Binks B.P. and Lee L T , J. Phys. II France 3 (1993) 1747

[3] Winsor P. A, Trans Faraday Soc. 44 (1948) 376

[4] Binks B P., Kellay H. and Meunier J., Europhys. Lett. 16 (1991) 53

[5] Kellay H., Meunier J and Binks B.P., Phys. Rev. Lett. 70 (1993) 1485

[6] Kellay H., Binks B P., Hendrikx Y., Lee L.T. and Meunier J , Adv. Coll. Interf. Sca 49 (1994) 85.

[7] Kellay H., Meunier J and Binks B.P, Phys. Rev. Lett. 69 (1992) 1220.

[8] Hendrikx Y., Kellay H. and Meunier J., Europhys. Lett. 25 (1994) 735

[9] Salager J.L., Bourrel M, Schechter R.S and Wade W.H., Soc Pet. Eng. J 19 (1979) 271.

[10] Oswald W, Z. Phys. Chem. 34 (1900) 495

[11] Meunier J., J. Phys. (Parrs) Lett 46 (1985) L-1005.

[12] Meunier J, Jérome B., Surfactant in Solution, Vol. 9, K.L. Mittal, Ed. (Plenum Publishing Corporation, 1989) p 463.

[13] Meunier J. and Binks B.P, Progr Coll Polym. Scı. 79 (1989) 178. 